\title{
Local Moduli Spaces in Analytic Geometry
}

\author{
By \\ Jürgen BINGENER*
}

\section{Introduction}

In this paper we formulate a general existence theorem for local moduli spaces for maps of graded complex spaces (3.2). From this result, which is proved in detail in [6], we obtain easily the existence of semiuniversal deformations of compact complex spaces, isolated singularities, coherent analytic sheaves with compact supports or isolated singularities, principal bundles over compact complex spaces and the existence of the Douady space and so on. Further applications are given in [5].

\section{$\S 1$. Graded Complex Spaces}

1.1. Let $A$ be a commutative ring. By a (positively) graded $A$-ringed space we understand an $A$-ringed space $X=\left(X, O_{X}\right)$ such that the structure sheaf of $X$ is an $N$-graded $A$-algebra $\mathcal{O}_{X}=\Perp_{i \in N}\left(\mathcal{O}_{X}\right)_{i}$. Then $\Gamma^{\prime}\left(U, \mathcal{O}_{X}\right):=\mathbb{H}_{i \in N} \Gamma\left(U,\left(\Theta_{X}\right)_{i}\right)$ is a graded $A$-algebra for every subset $U$ of $X$. In an obvious way one defines the notion of a morphism of graded $A$-ringed spaces.

Examples 1.2. (1) Let $X$ be a graded $A$-ringed space and $c$ an element of $N \cup\{\infty\}$. If we put $\left(\Theta_{X}\right)_{(\leqq c)}:=\Perp_{i \leqq c}\left(\Theta_{X}\right)_{i}$, then $X_{(\leqq c)}:=\left(X,\left(\Theta_{X}\right)_{(\leqq c)}\right)$ is again a graded $A$-ringed space, which is functorial in $X$. We will use the abbreviation $X_{0}:=X_{(\leq 9)}$.

(2) Let $X$ be an $A$-ringed space and $\mathscr{I}$ an $\mathcal{O}_{X}$-module. Then the trivial extension $X[\mathscr{F}]$ of $X$ by $\mathscr{I}$ is a graded $A$-ringed space in a natural way with $\left(\mathcal{O}_{X[\mathscr{F}]}\right)_{0}=\mathcal{O}_{X}$ and $\left(\mathcal{O}_{X[\mathcal{I}]}\right)_{1}=\mathscr{I}$.

(3) Let $X$ be an $A$-ringed space and $r=\left(r_{1}, \cdots, r_{m}\right)$ an element of $N_{+}^{m}$. There exists a unique graduation of the polynomial algebra $\mathcal{O}_{X}\left[w_{1}, \cdots, w_{m}\right]$ such that the variable $w_{j}$ has degree $r_{j}$ for $1 \leqq j \leqq m$. Hence $\left(X, \mathscr{O}_{X}\left[w_{1}, \cdots, w_{m}\right]\right)$

Communicated by M. Kashiwara, November 26, 1986.

* Fakultät für Mathematik der Universität, D-8400 Regensburg, Federal Republic of Germany. 
is a graded $A$-ringed space being denoted by $X(m ; r)$.

1.3. Let $X$ be a graded $A$-ringed space. By a graded $\mathcal{O}_{X}$-inodule we understand a module $\mathscr{I}$ over $\mathcal{O}_{X}$ endowed with a $\boldsymbol{Z}$-graduation $\mathscr{F}=\mathbb{\Perp}_{i \in Z} \mathscr{F}_{i}$ being compatible with the graduation of $\mathcal{O}_{X}$. Then $\mathscr{F}_{(\geqq c)}:=\Perp_{i \geqq c} \mathscr{F}_{i}$ resp. $\mathscr{I}_{(\leqq c)}:=$ $\Perp_{i \leqq c} \mathscr{F}_{i}$ is a graded submodule resp. quotient module of $\mathscr{F}$ for any element $c$ of $\boldsymbol{Z} \cup\{-\infty\}$.

1.4. A graded complex space is a graded $C$-ringed space $X$ such that the following properties hold: (1) $\left(X,\left(\Theta_{X}\right)_{0}\right)$ is a complex space. (2) $\mathcal{O}_{X}$ is an $\left(\Theta_{X}\right)_{0^{-}}$ algebra of finite presentation. (3) The homogeneous components $\left(\Theta_{X}\right)_{i}$ are coherent modules over $\left(\Theta_{X}\right)_{0}$.

The graded complex spaces form a full subcategory of the category of all graded $\boldsymbol{C}$-ringed spaces. It's easy to see that this category has finite fibre products.

By a graded pseudocomplex space we understand a graded $C$-ringed space $Z$ being locally isomorphic to a space of the form $\left(T, O_{X} \mid T\right)$, where $X$ is a graded complex space and $T$ is a closed subset of $X$. The graded pseudocomplex spaces again form a category with finite fibre products.

Examples 1.5. (1) Let $X$ be a graded complex space and $c$ an element of $N \cup\{\infty\}$. Then $X_{(\leqq c)}$ is again a graded complex space, which in case $c \in \mathbb{N}$ is a complex space at the same time.

(2) Let $X$ be a complex space. If we provide $\mathcal{O}_{X}$ with the trivial graduation, $X$ becomes a graded complex space. In this way we consider the category of complex spaces as a full subcategory of the category of graded complex spaces.

(3) Let $X$ be a complex space and $r=\left(r_{1}, \cdots, r_{m}\right)$ a tuple from $N_{+}^{m}$. Then $X(m ; r)$ is a graded complex space. In particular $C^{n}(m ; r)$ is a graded complex space.

1.6. Let $Z$ be a fixed graded complex space. We then define a groupoid $\mathrm{D}_{Z}$ over the category (Gan) of germs of analytic spaces as follows: An object of $\mathrm{D}_{Z}$ over a space germ $S=(S, 0)$ is a triple $f=(\mathscr{X} \rightarrow \mathscr{f}, K, L)$ consisting of $S$-flat graded complex spaces $\mathscr{X}, \mathscr{f}$ over $S \times Z$, closed subsets $K \leqq \mathscr{X}(0), L \subseteq \mathscr{J}(0)$ and an $S \times Z$-morphism $f: \mathscr{X} \rightarrow \mathscr{g}$ such that $f(K) \leqq L$. The morphisms in $\mathrm{D}_{Z}$ are defined in an obvious way.

For an object $f=(\mathscr{X} \stackrel{?}{\rightarrow} q, K, L)$ of $\mathrm{D}_{Z}(S)$, let $f(0)=(\mathscr{X}(0) \stackrel{f(0)}{\longrightarrow} \mathscr{Y}(0), K, L)$ denote the object of $\mathrm{D}_{Z}(\operatorname{Spec}(\boldsymbol{C}))$ obtained from $f$ by base change via the in- 
clusion of $\operatorname{Spec}(C)$ into $(S, 0)$. Further we have for any $c \in \mathbb{N}$ a natural functor

$$
\leqq c: \mathrm{D}_{Z} \longrightarrow \mathrm{D}_{Z(\leqq c)}
$$

of groupoids over (Gan) sending an object $(\mathcal{X} \rightarrow \rightarrow, K, L)$ to the object $\left(X_{(\leqq c)} \stackrel{\mu(\leqq c)}{\longrightarrow} \mathscr{Y}_{(\leqq c)}, K, L\right)$.

1.7. Let now $f=(X \stackrel{f}{\rightarrow} Y, K, L)$ be an object of $\mathrm{D}_{z}(\operatorname{Spec}(\mathbb{C}))$. Then

$$
\operatorname{Def}_{(f, K, L) / Z}^{\mathrm{gr}}:=\left(\mathrm{D}_{Z}\right)_{f}
$$

is again a groupoid over (Gan) such that $\operatorname{Def}_{(j, K, L) / Z}^{g r}(\operatorname{Spec}(C))$ consists of one object (up to isomorphism) admitting only one automorphism. We call the objects of that groupoid (graded) deformations of the map germ $(f, K, L):(X, K)$ $\rightarrow(Y, L)$ relative $Z$. Such a deformation over a space germ $S=(S, 0)$ is thus an object $f=\left(\mathscr{X} \rightarrow a_{0}, K, L\right)$ of $\mathrm{D}_{Z}$ over $S$ endowed with an isomorphism $f \stackrel{\sim}{\longrightarrow} f(0)$ in $\mathrm{D}_{Z}(\operatorname{Spec}(\boldsymbol{C}))$. The functor (1.6.1) induces a functor

$$
\left.\operatorname{Def}_{(f, K, L) / Z}^{g r} \longrightarrow \operatorname{Def}_{(f(\leqq c)}^{g r}, K, L\right) / Z_{(\leqq c)} \text {. }
$$

The fibre of this functor is a groupoid over (Gan), which we denote by

$$
\operatorname{Def}\left(\begin{array}{ll}
\mathrm{gr} \\
f, \geq c \geq 1 \\
, K, L) / Z
\end{array}\right.
$$

An object of this groupoid over a space germ $S$ consists of a deformation $f$ of $(f, K, L) / Z$ over $S$ endowed with an isomorphism $\left(f_{(\leqq c)}\right)_{S} \underset{\sim}{\longrightarrow}(\leqq c)$. In such a deformation, the homogeneous components of degree $\leqq c$ of $f$ are thus deformed in a trivial way. Finally we put $\operatorname{Def}_{(f, K, L, L) / Z}^{g r, \geq 0}:=\operatorname{Def}_{(f, K, L), Z \text {. }}^{g r}$

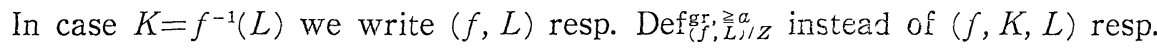

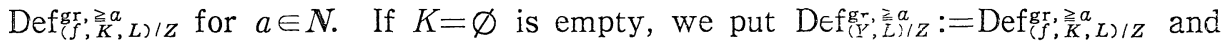
$\operatorname{Def}_{Y, Z}^{g_{1} \geq a}:=\operatorname{Def}_{(Y, \geq Y) / Z}^{g r} \geq a$ for $a \in \mathbb{N}$. Finally we suppress in case $Z=\operatorname{Spec}(C)$ the index $Z$ in the notations introduced above.

If $f: X \rightarrow Y$ is a map of complex spaces over a compler space $Z$, we shail consider $f$ as a $Z$-morphism of graded complex spaces with trivial graduation and omit the index "gr" in the above groupoids.

\section{§. Resolvents}

2.1. Let $X$ be a graded complex space. By a quasipoiyledron for $X$ we mean a triple $(P, \varphi, E)$ consisting of a compact subset $P$ of $X$, a closed embedding $\varphi: U \rightarrow V$ of graded complex spaces, where $U$ is an open subspace of $X$ and $V$ is an open subspace of a number space $C^{n^{\prime}}\left(n n^{\prime \prime} ; r\right)$, and an open special polycylinder $E \subseteq \mathbb{C}^{n^{\prime}}\left(n^{\prime \prime} ; r\right)$ with center $x_{0}$ and $\bar{E} \subseteq V$, such that $\varphi^{-1}(\bar{E})=P$ holds.

If $\varphi^{-1}\left(x_{0}\right)$ is non-empty, we say that $(P, \varphi, E)$ is a polyinedron for $X$ and 
call the unique point $x \in P$ such that $\varphi(x)=x_{0}$ the center of $(P, \varphi, E)$. In the following we write $P$ instead of $(P, \varphi, E)$ and denote $U$ resp. $V$ resp. $\varphi$ resp. $E$ by $U\langle P\rangle$ resp. $V\langle P\rangle$ resp. $\varphi\langle P\rangle$ resp. $E\langle P\rangle$.

2.2. Let now $f: X \rightarrow Y$ be a morphism of graded complex spaces. A quasipolyhedron for $f$ (or for $X$ relative $Y$ ) is a pair $(P, Q)$ consisting of a quasipolyhedron $P$ resp. $Q$ for $X$ resp. $Y$ having the following properties: (1) $f(U\langle P\rangle) \subseteq U\langle Q\rangle$. (2) $V\langle P\rangle$ is of the form $V\langle P\rangle=V\langle Q\rangle \times W$ with an open subspace $W$ of a number space $C^{l^{\prime}}\left(l^{\prime \prime} ; s\right)$, and the diagram

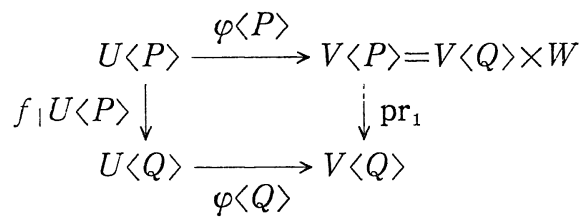

commutes. (3) $E\langle P\rangle$ is of the form $E\langle P\rangle=E\langle Q\rangle \times F$ with a polycylinder $F$ in $C^{l^{\prime}}\left(l^{\prime \prime} ; s\right)$. If $P$ and $Q$ are moreover polyhedra, we call $(P, Q)$ a polyhedron for $f$ (or for $X$ relative $Y$ ).

For any element $t$ of $] 0,1]$, the pair $\left(\left(\varphi\langle P\rangle, E\langle Q\rangle \times F^{(t)}\right), Q\right)$ is again a quasipolyhedron for $f$, which we call the shrinking of $(P, Q)$ with respect to $t$, and denote by $(P, Q)^{(t)}$. Suppose that $Q$ is a polyhedron with center $y$ and put $U:=U\langle P\rangle \cap X(y)$ and $P(y):=P \cap X(y)$. Then $\varphi\langle P\rangle$ induces a closed embedding $\varphi: U \rightarrow W$, and $(P(y), \varphi, F)$ is a quasipolyhedron for the fibre $X(y)$, which we denote by $(P, Q)(y)$.

2.3. Let now $\mathscr{P}=\left(P_{i}, Q\right)_{i \in I}$ be a family of polyhedra for $f: X \rightarrow Y$ over the same base polyhedron $Q$. The nerve of the family $\left(P_{i}\right)_{i \in I}$ of compact subsets of $X$ is a simplicial scheme over $I$, which we denote by $\operatorname{Ner}(\mathscr{P})$ and call the nerve of $\mathscr{L}$. The subset $|\mathscr{Q}|:=\bigcup_{i \in I} P_{i}$ of $X$ is called the support of $\mathscr{P}$.

For a tuple $t=\left(t_{\imath}\right)_{\imath \in I}$ of elements of $\left.] 0,1\right], \mathscr{L}^{(t)}:=\left(\left(P_{i}, Q\right)^{\left(t_{i}\right)}\right)_{\imath \in I}$ is again a family of polyhedra for $f$. If $s$ is a number such that $t_{i}=s$ for all $i$, we shall write $\mathscr{Q}^{(s)}$ instead of $\mathscr{Q}^{(t)}$. If $y$ denotes the center of $Q$, then $\mathscr{P}(y):=$ $\left(\left(P_{i}, Q\right)(y)\right)_{i \in I}$ is a family of polyhedra for the fibre $X(y)$ of $f$ in $y$. Finally we put

$$
n(\mathscr{D}):=\sup \left\{\operatorname{dim}(V\langle Q\rangle)+\operatorname{dim}\left(W_{\jmath}\right)+\sum_{i \in I} \operatorname{dim}\left(W_{i}\right): j \in I\right\} ;
$$

here $W_{i}$ is the graded complex space such that $V\left\langle P_{\imath}\right\rangle=V\langle Q\rangle \times W_{\imath}$.

We say that $\mathscr{Q}$ satisfies the polyhedral axiom, if: (1) There exists a tuple $t=\left(t_{i}\right)_{i \in I}$ of elements from $] 0,1\left[\operatorname{such}\right.$ that $\operatorname{Ner}(\mathscr{P})=\operatorname{Ner}\left(\mathscr{P}^{(t)}(y)\right)$. (2) For any subset $\left\{i_{0}, i_{1}\right\}$ in $\operatorname{Ner}(\mathscr{P})$, we have $P_{\iota_{0}} \cup P_{\imath_{1}} \leqq U\left\langle P_{\imath_{0}}\right\rangle \cap U\left\langle P_{i_{1}}\right\rangle$. 
2.4. Let $f: X \rightarrow Y$ be a morphism of graded complex spaces and $\mathscr{Q}=\left(P_{i}, Q\right)_{i \in I}$ a finite family of polyhedra for $f$. Suppose that $V\left\langle P_{i}\right\rangle=V\langle Q\rangle \times W_{i}, E\left\langle P_{2}\right\rangle=$ $E\langle Q\rangle \times F_{i}$ and $\varphi\left\langle P_{i}\right\rangle=\left(\rho_{i}, \tau_{i}\right) \quad$ with $\rho_{i}:=\varphi\langle Q\rangle \cdot\left(f \mid U\left\langle P_{i}\right\rangle\right)$ and morphisms $\tau_{i}: U\left\langle P_{i}\right\rangle \rightarrow W_{i}$. For an element $\alpha$ of $\Re:=\operatorname{Ner}(\mathscr{P})$ we abbreviate $U_{a}:=U_{\alpha}\langle\mathscr{P}\rangle$ : $=\bigcap_{i \in \alpha} U\left\langle P_{i}\right\rangle, W_{\alpha}:=W_{\alpha}\langle\mathscr{P}\rangle:=\prod_{i \in \alpha} W_{i}, V_{\alpha}:=V_{a}\langle\mathscr{P}\rangle=V\langle Q\rangle \times W_{a}$,

$$
\varphi_{\alpha}:=\varphi_{\alpha}\langle\mathscr{P}\rangle:=\left(\varphi\langle Q\rangle \cdot\left(f \mid U_{a}\right),\left(\tau_{i} \mid U_{a}\right)_{i \in \alpha}\right): U_{a} \longrightarrow V_{a},
$$

$F_{a}:=F_{\alpha}\langle\mathscr{P}\rangle:=\prod_{i \in \alpha} F_{i}, E_{\alpha}:=E_{a}\langle\mathscr{P}\rangle:=E\langle Q\rangle \times F_{a}$ and $P_{\alpha}:=\bigcap_{i \in a} P_{i} \leqq U_{\alpha}$. Then $\left(\left(P_{\alpha}, \varphi_{\alpha}, E_{\alpha}\right), Q\right)$ is a quasipolyhedron for $f$. Further $U_{*}:=U_{*}\langle\mathscr{P}\rangle:=\left(U_{a}\right)_{\alpha \in \Re}$ resp. $V_{*}:=V_{*}\langle\mathscr{P}\rangle:=\left(V_{\alpha}\right)_{\alpha \in \Re}$ is an $\Re$-coobject in the category of graded complex spaces over $U\langle Q\rangle$ resp. $V\langle Q\rangle$, and $\varphi:=\left(\varphi_{\alpha}\right)_{\alpha \in \Re}$ is a closed embedding of $U_{*}$ into $V_{*}$. Putting $\phi:=\varphi\langle Q\rangle$, the canonical diagram

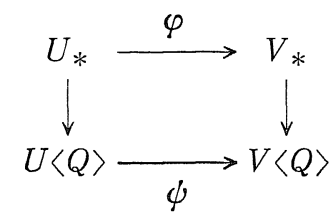

commutes. We abbreviate $F_{*}:=F_{*}\langle\mathscr{P}\rangle:=\left(F_{a}\right)_{\alpha \in g^{\circ} \text { 。 }}$ Then $\bar{F}_{*}:=\left(\bar{F}_{a}\right)_{a \in \mathfrak{l}}$ and $P_{*}$ : $=P_{*}\langle\mathscr{P}\rangle:=\left\langle P_{a}\right)_{a \in \Re}$ are in a natural way $\Re$-coobjects in the category of graded pseudocomplex spaces. With these notations we obtain from (2.4.1) the following canonical diagram

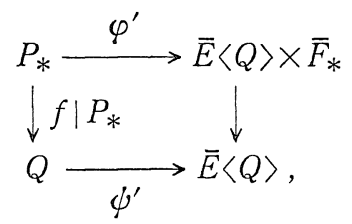

where the horizontal arrows are closed embeddings.

2.5. Let the assumptions and notations be as in 2.4. By a sheaf resolvent for $f$ (or for $X$ relative $Y$ ) with respect to $\mathscr{P}$ we understand a pair $(\mathscr{B}, \mathscr{R}$ ) consisting of a $g$-finite free graded $D G$-Algebra $\mathscr{B}=(\mathscr{B}, s)$ over $\mathcal{O}_{\bar{E}\langle Q\rangle}$ with $\mathscr{B}^{0}=$ $\mathcal{O}_{\bar{E}\langle Q\rangle}$ and a $g$-finite free graded $D G$-algebra $\mathscr{R}=(\mathscr{R}, s)$ over

$$
\mathscr{B} \otimes_{\mathcal{O}_{\bar{E}}\langle Q\rangle} \mathcal{O}_{\bar{E}\langle Q\rangle \times \bar{F}_{*}}
$$

with $\mathscr{R}^{0}=\mathcal{O}_{\bar{E}\langle Q\rangle \times \bar{F}_{*}}$ such that $\mathscr{B}$ resp. $\mathscr{R}$ is a resolution of $\phi_{*}^{\prime}\left(\mathcal{O}_{Y} \mid Q\right)$ resp. $\varphi_{*}^{\prime}\left(\Theta_{X} \mid P_{*}\right)$. One can easily show that for a given $\mathscr{Q}$ there always exists a sheaf resolvent for $f$ with respect to $\mathscr{Q}$.

2.6. Let now $X$ and $Y$ be two graded complex spaces over a complex space $Z$ and let $f: X \rightarrow Y$ be a $Z$-morphism. By a polyhedron for $f / Z$ we understand a triple $(P, Q, M)$ such that $(P, Q)$ resp. $(Q, M)$ is a polyhedron for $f$ resp. for 
$Y$ relative $Z$. The phrases and notations introduced in 2.3 carry over in an obvious way to families of polyhedra for $f / Z$.

Let $\mathscr{P}=\left(P_{i}, Q, M\right)_{i \in I}$ be a finite family of polyhedra for $f / Z$. Then $\mathscr{P}$ induces in a natural way a commutative diagram

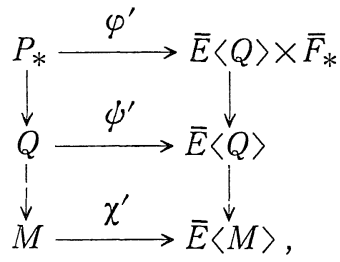

see (2.4.2). By a sheaf resolvent for $f / Z$ with respect to $\mathscr{L}$ we understand a pair consisting of a sheaf resolvent $A \rightarrow \mathscr{B}$ for $Y$ relative $Z$ with respect to $(Q, M)$ and a sheaf resolvent $\mathscr{B} \rightarrow \mathbb{R}$ for $f$ with respect to $\left(P_{i}, Q\right)_{i \in I}$.

2.7. Let $Z, f: X \rightarrow Y$ and $\mathscr{Q}=\left(P_{i}, Q, M\right)_{i \in I}$ be as in 2.6. Further let $\Re:=$ $\operatorname{Ner}(\mathscr{P})$ be the nerve of $\mathscr{L}$, and let $\mathcal{A} \rightarrow \mathscr{B} \rightarrow \mathscr{R}$ be a sheaf resolvent for $f / Z$ with respect to $\mathscr{Q}$, and put $S:=E\langle Q\rangle_{0}$. Moreover let $c$ resp. $d$ be a fixed number from $N$ resp. $Z_{\leqq 0}$. By ${ }^{c} \mathscr{M}_{\mathscr{R} \mid \mathscr{B}}$ resp. ${ }^{c} \mathscr{M}_{\mathscr{B} \mid \mathcal{A}}$ resp. ${ }^{c} \mathscr{M}_{\mathscr{R} \mid \mathscr{B}, \mathcal{A}}$ we denote the $D G$ module over $\mathcal{O}_{S}$, whose sections over an open subset $V$ of $S$ are the compatible families of homomorphisms from

$$
\prod_{a \in \mathcal{R}} \operatorname{Hom}_{\mathcal{R}_{\alpha} \mid V \times F_{\alpha}}\left(\Omega_{\mathcal{R}_{\alpha}|\mathfrak{B}|} V \times F_{\alpha},\left(\mathscr{R}_{\alpha}\right)_{(\geqq c)} \mid V \times F_{\alpha}\right)
$$

resp.

resp.

$$
\operatorname{Hom}_{\mathscr{B} \mid V}\left(\Omega_{\mathscr{B} / \mathfrak{A}}\left|V, \mathscr{B}_{(\succeq c)}\right| V\right)
$$

$$
\prod_{\alpha \in \mathscr{R}} \operatorname{Hom}_{\mathbb{R}_{\alpha}^{\prime} V \times F_{\alpha}}\left(\Omega_{\mathcal{R}_{\alpha^{\prime}} \mathfrak{A}}\left|V \times F_{\alpha},\left(\mathscr{R}_{\alpha}\right)_{(\geqq c)}\right| V \times F_{\alpha}\right) \times \Gamma\left(V,{ }^{c} \mathscr{M}_{\mathscr{B} \mid \mathcal{A}}\right),
$$

the differential being the map $u \mapsto[s, u]$. Further let $\mathscr{F}^{d}\left({ }^{c} \mathscr{M}_{\mathscr{R} \mid \mathscr{B}}\right)$ resp. $\mathscr{I}^{d}\left({ }^{c} \mathscr{M}_{\mathscr{B} \mid \mathcal{A}}\right)$

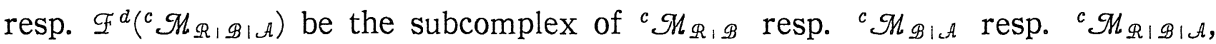
whose sections over an open subset $V$ of $S$ are the families vanishing on the $\Omega_{\mathscr{R} / \mathscr{B}}^{\nu} \mid V \times F_{*}$ resp. $\Omega_{\mathscr{B} / \mathcal{A}}^{\nu} \mid V$ resp. on the $\Omega_{\mathscr{R} / \mathcal{A}}^{\nu} \mid V \times F_{*}$ and $\Omega_{\mathscr{B} / \mathcal{A}}^{\nu} \mid V$ for $\nu \geqq d$. Then

$$
\begin{aligned}
& \text { (c,d) } \mathscr{M}_{\mathscr{R} \mid \mathcal{B}}:={ }^{c} \mathscr{M}_{\mathbb{R} \mid \mathcal{B}} / \mathscr{F}^{d}\left({ }^{c} \mathscr{M}_{\mathscr{R} \mid \mathcal{B}}\right) \text {, } \\
& { }^{(c, d)} \mathscr{M}_{\mathscr{B} \mid \mathcal{A}}:={ }^{c} \mathscr{M}_{\mathscr{B} \mid \mathcal{A}} / \mathscr{F}^{d}\left({ }^{c} \mathscr{M}_{\mathscr{B} \mid \mathcal{A}}\right) \text {, } \\
& \text { (c, d) } \mathscr{M}_{\mathbb{R}|\mathcal{B}| \mathcal{A}}:={ }^{c} \mathscr{M}_{\mathcal{R}|\mathcal{B}| \mathcal{A}} / \mathscr{F}^{d}\left({ }^{c} \mathcal{M}_{\mathcal{R}|\mathcal{B}| A}\right)
\end{aligned}
$$

are in a natural way complexes in $\mathbf{K}\left(\mathrm{PO}_{1,1}\left(\Theta_{S}\right)\right)$ having nuclear free components (see [3], 2.3, 3.4 and 3.6), and the canonical sequence

$$
0 \longrightarrow{ }^{(c, d)} \mathscr{M}_{\mathscr{R} \mid \mathscr{B}} \longrightarrow^{(c, d)} \mathscr{M}_{\mathscr{R}|\mathscr{B}| \mathcal{A}} \longrightarrow{ }^{(c, d)} \mathscr{M}_{\mathscr{B} \mid \mathcal{A}} \longrightarrow 0
$$

of complexes is exact and splits naturally in $\mathrm{G}\left(\mathrm{PO}_{1,1}\left(\mathcal{O}_{S}\right)\right)$. 


\section{$\S 3$. Versal Deformations of Maps of Graded Complex Spaces}

3.1. Let $X$ and $Y$ be two graded complex spaces over a graded complex space $Z, f: X \rightarrow Y$ a $Z$-morphism, $c \in \mathbb{N}$ a natural number, $T:=\operatorname{Supp}\left(\left(\Theta_{X}\right)_{(\geqq c)}\right)$, $L \subseteq Y$ a closed subset and $K:=f^{-1}(L) \cap T$.

Theorem 3.2. Let the assumptions and notations be as in 3.1. Moreover suppose that:

(1) $T$ is proper over $Y$, and $L$ is finite.

(2) The C-vector space $\overline{\operatorname{Def}_{(f, K, L}^{g r}, L, Z}(D)$ of isomorphism classes of deformations of $(f, K, L)$ in $\operatorname{Def}_{(f, \bar{K}, \bar{K}, L) / Z}^{\mathrm{gr}}$ over the double point $D$ is finite dimensional.

Then the germ $(f, K, L):(X, K) \rightarrow(Y, L)$ has a semiuniversal deformation in the groupoid $\left.\operatorname{Def}_{(f, \mathbb{K}}^{\mathrm{gr}}, \frac{2}{K}, L\right) / Z$ over $(\mathrm{Gan})$.

Sketch of proof. We may assume that $L=\{y\}$ consists of one point. Then there exists a family $\mathscr{Q}=\left(P_{i}, Q, M\right)_{\imath \in I}$ of polyhedra for $f / Z$ and an element $z$ of $] 0,1[$ such that the following properties hold: (1) $\mathcal{P}$ is finite and satisfies the polyhedral axiom. (2) $y$ is the center of $Q$. (3) We have $\operatorname{Ner}(\mathscr{P})=$ $\operatorname{Ner}\left(\mathscr{P}^{(z)}(y)\right)$. (4) $f^{-1}(Q) \cap T$ is contained in $\left|\mathscr{Q}^{(z)}\right|$.

Let $A \rightarrow \mathscr{B} \rightarrow \mathscr{R}$ be a sheaf resolvent for $f / Z$ with respect to $\mathscr{Q}$ and $d \leqq 0$ an integer with $d \leqq-n(\mathscr{Q})-7$. We put $S:=E\langle Q\rangle_{0}$ and

$$
\begin{aligned}
& E:=\Gamma\left(S,{ }^{(c, d)} \mathscr{M}_{\mathscr{Q} \mid \mathcal{B}}\right), \\
& \left.F:=\Gamma\left(S,{ }^{(c)} d\right) \mathcal{M}_{\mathbb{R}|\mathcal{B}| \mathcal{A}}\right), \\
& G:=\Gamma\left(S,{ }^{(c, d)} \mathcal{M}_{\mathscr{B} \mid \mathcal{A}}\right)
\end{aligned}
$$

for abbreviation, see 2.7. Then $E, F$ and $G$ are in a natural way complexes in $\mathbb{K}\left(\mathrm{PO}_{1}(\boldsymbol{C})\right)$, and we have a canonical exact sequence

$$
0 \longrightarrow E \longrightarrow F \longrightarrow G \longrightarrow 0,
$$

which splits in $\mathrm{G}\left(\mathrm{PO}_{1}(\mathbb{C})\right.$ ). If we can show that the complex $F^{(0,2)}$ splits in $\mathbb{K}(\mathrm{PO}(\boldsymbol{C})$ ) (for a suitable choice of $\mathscr{Q})$, then the assertion of 3.2 follows from the quotient theorem ([4] 4.3). For this purpose it suffices to prove the following :

(a) The complex $E^{(0,3)}$ splits in $\mathbb{K}(\mathrm{PO}(\mathbb{C}))$.

(b) The complex $G^{(-1,2)}$ splits in $\mathbb{K}(\mathrm{PO}(\mathbb{C})$ ).

(c) The coboundary maps $\delta^{2}: \mathrm{H}^{\imath}(G) \rightarrow \mathrm{H}^{\imath+1}(E)$ split in $\mathrm{PO}(\mathbb{C})$ for $i=0,1$.

The properties (b) and (c) follow (for a suitable choice of $Q$ and $M$ ) from the existence of privileged neighborhoods.

The property (a) is (for a suitable choice of $\mathscr{L}$ ) a consequence of the split- 
ting criteria [3], 2.4, 4.2, 4.3 (1). Here the fundamental problem is the verification of the assumption (2) of loc. cit. 2.4. In order to do this, one first shows, that the property (a) is independent of the choice of the resolvent $R$. This is the assertion of the theorem of the homotopy invariance of the (co)tangent complex, see [16] and [6] (III 4.15). The actual verification will be made then only for very special, so called admissible resolvents. Here the theorem on the "Nullhomotopie des Čech-Komplexes einer Auflösung" ([6] (II 5.3)) is an essential ingredient.

Remark 3.3. Generalizing the methods used here, one should be able to show that 3.2 remains valid, if one replaces the map $f: X \rightarrow Y$ by an $n$-chain

$$
X^{(n)} \stackrel{f^{(n-1)}}{\longrightarrow} X^{(n-1)} \longrightarrow \cdots \longrightarrow X^{(2)} \stackrel{f^{(1)}}{\longrightarrow} X^{(1)}
$$

of graded complex spaces over $Z$ (with $n \in N_{+}$). Further 3.2 should be true also under suitable concavity assumptions on $T$. $I$ hope to come back to this at another place.

\section{$\S 4$. Consequences}

If we apply 3.2 to a map of complex spaces and $c=0$, we get the following result, which $I$ obtained already in 1983.

Theorem 4.1. Let $X$ and $Y$ be complex spaces over a complex space $Z$, $f: X \rightarrow Y$ a proper $Z$-morphism and let $L \subseteq Y$ be a finite subset. If the $C$-vector space $\overline{\operatorname{Def}}_{(f, L) / Z}(D)$ of isomorphism classes of deformations of $(f, L)$ over the double point is finite dimensional, the germ $(f, L):\left(X, f^{-1}(L)\right) \rightarrow(Y, L)$ has a somiuniversal deformation in the groupoid $\operatorname{Def}_{(f, L) / Z \text {. }}$

Putting $Y=Z=\operatorname{Spec}(\boldsymbol{C})$ resp. $X=\varnothing$ and $Z=\operatorname{Spec}(\boldsymbol{C})$ in 4.1 , we obtain the next two propositions.

Theorem 4.2 (Douady [10], Grauert [14], Forster-Knorr [12], Palamodov [17]). Let $X$ be a compact complex space. Then $X$ has a semiuniversal deformation.

Theorem 4.3 (Donin [7], Grauert [13], Pourcin [21]). Let $Y$ be a complex space and $L \subseteq Y$ a finite subset. If $\overline{\operatorname{Def}}_{(Y, L)}(D)$ is finite dimensional, the germ $(Y, L)$ has a semiuniversal deformation.

The well known existence theorems for deformations of coherent sheaves are contained in 3.2 , too:

Theorem 4.4 (Siu-Trautmann [23]). Let $X$ be a complex space and $F$ a 
coherent $\mathcal{O}_{X}$-module with compact support. Then I has a semiuniversal deforma. tion.

This follows from 3.2, applied to $X=X[\mathscr{F}], Y=Z=\operatorname{Spec}(\mathbb{C})$ and $c=1$.

Theorem 4.5 (Trautmann [24]). Let $Y$ be a complex space, $\mathcal{G}$ a coherent $\mathcal{O}_{Y^{-}}$ module and let $L \subseteq Y$ be a finite subset such that $\operatorname{Supp}\left(\mathcal{E}_{x+t_{\mathcal{O}}^{1}}(\mathcal{G}, \mathcal{G})\right) \subseteq L$. Then the germ $(G, L)$ has a semiuniversal deformation.

This also follows from 3.2, applied to $X=\varnothing, Y=Y[\mathcal{G}], Z=\operatorname{Spec}(\mathbb{C})$ and $c=1$. - Using [2], one can deduce from 4.4 the following result:

Theorem 4.6 (Douady-Pourcin $[9,20]$ )。 Let $f: X \rightarrow S$ be a separated holomorphic map and $\mathscr{T}$ a coherent $\mathcal{O}_{X}$-module. Then the functor $\mathrm{Q}_{I / X / S}$ of Grothendieck is representable by a separated complex space over $S$.

In [19] it was shown, that the following theorem is a consequence of 4.4 and 4.6, and hence altogether also of 3.2.

Theorem 4.7. (Ponomarev [19], Donin [8]). Let $X$ be a compact complex space, $G$ a complex Lie group and $P \rightarrow X$ a G-principal bundle over $X$. Then $P$ has a semiuniversal deformation.

Remarks 4.8. (1) In [6] we also show a local version of 3.2 , containing as special cases as well the theorem of Retakh on versal deformations of analytic map germs, which was announced in [22] and proved in detail in [11], as the propositions $4.3,4.5$.

(2) Let $S$ be a fixed analytic space germ. Then both theorem 3.2 and its corollaries remain $\mathrm{m} . \mathrm{m}$. true, if we replace the category (Gan) of analytic space germs by the category $(\mathrm{Gan} / S$ ) of space germs over $S$ (compare also [4], 4.6 (2)). I will give the details of this generalization to the "relative case" at another place.

(3) In a subsequent paper I will show that one can construct versal deformations of complex spaces (and maps) with a group action using a suitable generalization of the methods employed in this paper. In this approach the description of equivariant deformations given in [15] plays an essential part.

(4) A fully satisfactory treatment of analytic deformation theory seems only be possible within the frame of the so-called anticommutative (graded) complex spaces (see [6], (VI 7)). These objects, which appear here only in disguise of the resolvents, are known in a somewhat more special form under the name "complex superspaces" since a while. I intend to give the details in 
subsequent papers.

\section{References}

[1] Bingener, J., Offenheit der Versalitat in der analytischen Geometrie, Math. Z., 173 (1980), 241-281.

[2] - Darstellbarkeitskriterien für analytische Funktoren, Ann. Sci. ENS, 13 (1980), 317-347.

[3] - Splitting theorems for PO-complexes, Publ. RIMS, Kyoto Univ., 23 (1987), 527-534.

[4] —- Analytic moduli spaces as orbit spaces, Publ. RIMS, Kyoto Liniv., 23 (1987), 535-542.

[5] — L Local moduli for strictly pseudoconvex spaces, Publ. RIMS, Kyoto Univ., 23 (1987), 553-558.

[6] Bingener, J., Kosarew, S., Lokale Modulräume in der analytischen Geometrie, Vieweg-Verlag, Braunschweig/Wiesbaden (1987).

[7] Donin, I. F., Complete families of deformations of germs of complex spaces, Math. USSR Sb., 18 (1972), 397-406.

[8] — Construction of a versal family of deformations for holomorphic bundles over a compact complex space, Math. USSR Sb., 23 (1974), 405-416.

[9] Douady, A., Le problème des modules pour les sous-espaces analytiques compacts d'un espace analytique donné, Ann. Inst. Fourier, 16 (1966), 1-95.

[10] — Le problème des modules locaux pour les espaces $\boldsymbol{C}$-analytiques com pactes, Ann. Sci. ENS, 7 (1974), 569-602.

[11] Flenner, H., Über Deformationen holomorpher Abbildungen, Habilitationsschrift, Osnabrück 1978.

[12] Forster, O., Knorr, K., Konstrukiion verseller Familien kompakter komplexer Räume, Lecture Notes in Mathematics 705, Springer-Verlag, Berlin-Heidelberg-New York (1979).

[13] Grauert, H., Über die Deformationen isolierter Singularitäten analytischer Mengen, Invent. Math., 15 (1972), 171-198.

[14] — - Der Satz von Kuranishi für kompakte komplexe Räume, Invent. Math., 25 (1974), 107-142.

[15] Illusie, L., Complexe Cotangent et Déformations II, Lecture Notes in Mathematics 283, Springer-Verlag, Berlin-Heidelberg-New York (1972).

[16] Kosarew, S., The homotopical invariance of (co-) tangent complexes, to appear.

[17] Palamodov, V.P., Deformations of complex spaces, Russian Math. Surveys, 31 (1976), 129-197.

[18] - The tangent complex of an analytic space, AMS Translations, 122 (1984), 119-171.

[19] Ponomarev, D. A., Deformations of smooth analytic fiberrings on spaces with resolvable singularities, Math. USSR Sb., 17 (1972), 617-623.

[20] Pourcin, G., Théorème de Douady au-dessus de S, Ann. Sc. Norm. Pisa, 23 (1969), 451-459.

[21] - Déformation de singularités isolées, Astérisque, 16 (1974), 161-173.

[22] Retakh, V.S., The "universal" deformation of germs of holomorphic mappings, Funk. Anal. Appl., 8 (1974), 80-81.

[23] Siu, Y.-T., Trautmann, G., Deformations of coherent analytic sheaves with compact supports, Memoirs Am. Math. Soc., 238 (1981).

[21] Trautmann, G., Deformationen von isolierten Singularitäten kohärenter analytischer Garhen, Math. Ann., 223 (1976), 71-89. 